\title{
First Survey for Detecting the Presence of Human Herpesvirus 8 Infection (HHV-8) in Maputo, Mozambique
}

\author{
Adele Caterino-de-Araujo ${ }^{1}$, Rolanda Carmen Rafael Manuel $^{2}$, Rosana Del Bianco ${ }^{3}$, Elizabeth Santos-Fortuna ${ }^{1}$, Mariana \\ Cavalheiro Magri ${ }^{1}$, Joyce Matie Kinoshita Silva ${ }^{1}$ and Rui Bastos ${ }^{2}$ \\ ${ }^{1}$ Immunology Department, Instituto Adolfo Lutz, Secretary of Health of São Paulo, São Paulo, SP., Brazil; ${ }^{2}$ Dermatology Unit, Central Hospital \\ of Maputo, Eduardo Mondlaine University, Maputo, Mozambique; ${ }^{3}$ AIDS Reference Centre of São Paulo, Secretary of Health of São Paulo, \\ São Paulo, SP., Brazil
}

\begin{abstract}
Human herpesvirus 8 (HHV-8), the etiological agent of Kaposi's sarcoma (KS), is endemic in parts of the subSaharan, and KS has increased concomitantly with the HIV/AIDS epidemic. In Mozambique (MZ), no data concerning $\mathrm{HHV}-8$ infection was available, thus the main of this work was to investigate, for the first time, the presence of $\mathrm{HHV}$ 8 infection in Maputo, MZ. Latent and lytic HHV-8-specific antibodies were assessed in blood samples from 189 individuals from the Central Hospital of Maputo, MZ, using "in-house” immunofluorescence assays conducted in São Paulo, Brazil. The results obtained were analyzed according to socio-demographic and clinical variables using the Chi-square test and logistic regression. An HHV-8 seropositivity of $\mathbf{1 . 8 \%}$ and $\mathbf{9 . 7 \%}$ was detected among 57 medical students and 31 individuals from the staff, respectively, in contrast to $16.4 \%$ detected among 67 out-patients. Concerning 34 hospitalized patients from the Dermatology Unit, 47.1\% were HHV-8-seropositive overall, while the rate was $\mathbf{8 5 . 7 \%}$ among $\mathrm{KS}$ patients. The present survey, conducted in Maputo, MZ, demonstrates great variation in HHV-8 infection frequencies depending on the group analyzed and epidemiological variables. An association between HHV-8 seropositivity and male gender (OR 5.72), the central origin of patients (OR 5.33), blood transfusions (OR 3.25), and KS (OR 24.0) was detected among hospitalized patients, and primary school (OR 7.18) and HIV-1 infection (OR 8.76) among out-patients.
\end{abstract}

Key-Words: Human herpesvirus 8 (HHV-8), serology, Mozambique, frequency, Kaposi’s sarcoma.

Human herpesvirus 8 (HHV-8) is considered as the etiological agent of all forms of Kaposi's sarcoma (KS), a tumor that frequently occurs during HIV infection (epidemic KS [AIDS-KS]) transplant recipients (iatrogenic KS), elderly men of Mediterranean and Middle Eastern origin (classic KS), and inhabitants of eastern and central Africa (endemic KS) [1,2].

During the last decades on African continent, AIDS has had a profound impact on adult and child mortality, reducing life expectancy by 10-15 years, mostly in sub-Saharan countries [3]. This high rate of mortality is attributed to opportunistic infections and neoplasms, with an emphasis on tuberculosis and KS.

Although the incidence of KS depends on the prevalence of HHV-8, several factors have been associated with disease development and, in many African countries, KS has risen dramatically since the onset of the AIDS epidemic [4,5]. Interestingly, the introduction of highly active antiretroviral therapy (HAART) reverted the increase in KS in wealthier

Received on 6 April 2009; revised 8 June 2009.

Address for correspondence: Dr. Adele Caterino-de-Araujo, Immunology Department, Instituto Adolfo Lutz, Av. Dr. Arnaldo, 355, $11^{\circ}$ andar, 01246-902, São Paulo, S.P., Brazil. Phone/Fax: +55-11-30682898, email: caterino@usp.br, caterino@ial.sp.gov.br. Financial support: Ministério da Ciência e Tecnologia/Conselho Nacional de Desenvolvimento Científico e Tecnológico (MCT/CNPq), Brasil, (PROAFRICA \# 490179/2006-1 and \# 490452/2007-8), fellowship to ACA (PD \# 304372/2006-4), and to MCM (Master in Pharmacy student). JMKS is a graduate student supported by the Secretary of Heath of São Paulo. Presented in part at the $13^{\text {th }}$ International Congress on Infectious Diseases, 19-22 June, 2008, Kuala Lumpur, Malasya (Abstract \# 16004).

The Brazilian Journal of Infectious Diseases 2009;13(3):200-202. (C) 2009 by The Brazilian Journal of Infectious Diseases and Contexto Publishing. All rights reserved. countries [5], but because of limited access to antiretroviral therapies in Africa, KS continues to increase in this geographic area. The exact mechanism by which HAART controls KS progression is unknown, but it has been speculated that the control of HIV viral load by HAART and consequent low Tat protein production, which is thought to be involved in HHV8 entry into the cell, leads to a decrease in virus spreading and HHV-8-associated diseases. In addition, reconstitution of the immune system after HAART could improve and/or prevent KS lesions [5,6]. Thus, knowledge of the prevalence of HHV8, mostly among HIV-1-infected patients is necessary for the best control and management strategies for patients, as well as for controlling transmission/acquisition of stigmatizing and lethal AIDS-KS in Africa.

Until now, no study examining HHV-8 infection has been conducted in Mozambique (MZ), a country that is experiencing an AIDS epidemic (16.0\% [14.0\% to $17.0 \%$ ] of individuals were living with HIV in 2007: 9.0\% in the northern, $18.0 \%$ in the central, and $21.0 \%$ in the southern region of the country) [1], and is bordered in the northern and central regions by countries where KS is endemic [4]. Thus, an exploratory mission from Brazil supported by the Ministry of Science and Technology was conducted in Maputo, MZ with the aim of performing a preliminary survey on the occurrence of HHV-8 infection in this geographic area.

\section{Material and Methods}

The mission consisted of a visit by two Brazilian physicians to the Central Hospital of Maputo, University Eduardo Mondlaine, Maputo, MZ, who joined with local physicians and together established a plan for selecting individuals who could be included in this preliminary survey, 
as well as more appropriate demographic, clinical and laboratorial variables for analysis. A questionnaire containing information on socio-demographic characteristics of individuals such as gender, age, geographic origin, house location, number of household persons, number of siblings, household electricity, household tap water, marital status, occupation, and years of education was generated. Information regarding prior blood transfusions, number of sexual partners in the last months, prior exposure to sexually transmitted diseases (STDs), partners with AIDS, hepatitis, use of illicit drugs, HIV status, and KS were included. All participants were interviewed, answered the questionnaire, and signed the informed consent form before blood collection was performed. The study was approved by the Ethics Committees of Brazilian Institutions, and by the Ethics Committees of the Ministry of Health of Mozambique and Brazil.

Blood samples were collected on filter paper from 189 individuals: 57 students from the medical school (Group 1), 31 individuals from the staff (Group 2), 67 out-patients (in ambulatory assistance at the Dermatology Department, Group 3), and 34 hospitalized patients from the Dermatology Unit (Group 4). Subsequently, one physician from MZ came to Brazil in order to update her knowledge of AIDS-KS at a Reference Centre of AIDS in São Paulo. Latent and lytic HHV8 antibodies were assessed in eluated blood using "in house" indirect immunofluorescence assays (IFA-LANA and IFI-Lytic) at the Instituto Adolfo Lutz, São Paulo, Brazil as previously described. Briefly, cells of the BCBL-1 line latently infected with HHV-8 or stimulated with tetradecanoyl phorbol ester acetate (TPA) were used to prepare slides for IFA-LANA and IFA-Lytic, respectively. Samples were eluated from dried blood punches of $6 \mathrm{~mm}$ diameter in $200 \mu \mathrm{L}$ of PBS (dilution 1:40), and then adjusted to $1: 50,1: 100$, and 1:200 in PBS/skim milk. Samples were considered HHV-8 seropositive when the results showed reactivity in at least one IFA (LANA or Lytic) in three dilutions employed. The sensitivity and specificity of the assays were confirmed in KS-AIDS patients and healthy persons from São Paulo [8]. Statistical analysis of sociodemographic and clinical variables, and HHV-8 serological results in each Group were conducted using the Chi-square test and a p level $\leq 0.05$ was considered statistically significant. Logistic regression was employed for calculating the odds ratio (OR) and 95\% confidence intervals (CI).

\section{Results}

Table 1 presents the HHV-8 serological results from each Group analyzed, and Table 2 shows statistically significant associations. The results obtained showed great variation in HHV-8 frequencies of infection in Maputo (south of MZ), with low frequencies among healthy individuals (Group 1 and 2 ) and high frequencies among hospitalized patients (Group 4). However, the best Groups for analysis were Group 3 and 4 , comprising individuals of several ages and different sociodemographic statuses (data not shown); among them, significant associations between HHV-8 seropositivity and male gender, the central origin of patients, primary school, prior blood transfusions, KS and HIV-1 infection were detected (Table 2).

\section{Discussion}

The results obtained could suggest that Maputo is not an endemic area of HHV-8, but we cannot exclude bias in these results since the individuals analyzed were not representative

Table 1. HHV-8 serological results using IFA-LANA and IFA-Lytic according to the group analyzed.

\begin{tabular}{lccc}
\hline Group & HHV-8 + & HHV-8 - & Total \\
\hline N=189 & $\mathrm{N}(\%)$ & $\mathrm{N}(\%)$ & $\mathrm{N}$ \\
G1 - students & $1(1.8)$ & $56(98.2)$ & 57 \\
G2 - staff & $3(9.7)$ & $28(90.3)$ & 31 \\
G3- out-patients & $11(16.4)$ & $56(83.6)$ & 67 \\
G4- hospitalized patients & $16(47.1)$ & $18(52.9)$ & 34 \\
\hline
\end{tabular}

N: number of individuals; G: Group; + denote sera that gave a positive result at least once. IFA-LANA or IFA-Lytic; - denote sera that were negative for HHV-8 serology. Chi-square test with statistical significance: G1 vs G3 p=0.006; G1 vs G4 p=0.000; G2 vs G4 p=0.001; G3 vs G4 p=0.001.

Table 2. Significant associations between socio-demographic and clinical variables and HHV-8 positivity in each Group analyzed.

\begin{tabular}{llccc}
\hline Variable & vs & Group & Chi-square & OR (95\% CI) \\
\hline Gender & G4 & male & $\mathrm{p}=0.017$ & $5.72(1.06-34.02)$ \\
Geographic origin & G4 & central & $\mathrm{p}=0.160$ & $5.33(0.71-49.11)$ \\
Education & G3 & primary & $\mathrm{p}=0.019$ & $7.18(1.47-37.52)$ \\
Blood transfusion & G4 & yes & $\mathrm{p}=0.036$ & $3.25(0.59-19.08)$ \\
Kaposi's sarcoma & G4 & yes & $\mathrm{p}=0.000$ & $24.0(2.99-257.38)$ \\
HIV & G & yes & $\mathrm{p}=0.009$ & $8.76(1.51-65.78)$ \\
\hline
\end{tabular}

G3: out-patients; G4: hospitalized patients. Chi-square test ( $\mathrm{p} \leq 0.05$ was considered statistically significant). Odds ratio (OR) and 95\% Confidence Intervals (CI) were calculated using logistic regression. 
of the general population of Maputo or other regions of the country.

Nevertheless, concerning gender, the majority of studies worldwide have demonstrated more cases of KS among males, and female hormones have been suggested to be protective factors in HHV-8 transmission/acquisition [1,2,4,5]. With regard to the central origin of HHV-8-infected individuals detected in the present study, this result is not surprising because of the vicinity of this region to HHV-8- and KS-endemic countries, such as Tanzania, Malawi, Zambia, and Zimbabwe [4]. Interestingly, HIV was introduced and spread in MZ via the northern and central regions, and is now increasing in the south [7,9]; thus we can speculate that an increase in the epidemic form of KS could occur in the south of MZ in the future. In support of this hypothesis, the highest rate of HHV8-seropositivity in the present study was detected among out-patients and hospitalized patients, most of which were HIV-1-infected, and had originated in the central region of MZ.

The association between HHV-8 infection and a low education level (primary school) of the individuals could suggest that poor household conditions facilitate virus transmission/acquisition. In support of this finding, some studies conducted in Africa demonstrated poor sanitary and hygienic practices as well as lack of tap water and low socioeconomic status as risk factors for HHV-8 transmission/ acquisition [10,11]. In addition, segments of DNA/HHV-8 were detected in saliva and urine, which implicate these specimens as potential routes of virus transmission [5,12]. Of note, although not statistically significant, several individuals enrolled in the present study mentioned lack of electricity and tap water at home. Thus, we can speculate that HHV-8 infection may be preventable if sanitary conditions and the population education level were improved in Africa.

The association between HHV-8 infection and previous blood transfusions are in agreement with the finding of the presence of virus in blood samples [5]. No association between HHV-8 and exposure to STDs, use of illicit drugs, hepatitis, or other variables was detected, but we could not exclude participant omission or lack of knowledge regarding this information. Concerning the significant association between $\mathrm{KS}$ and HIV, the results are in accordance with the literature as well as our previous studies confirming HHV-8 infection in KS patients, most of whom were HIV/AIDS patients [6,8,12,13]. Although the number of individuals analyzed was small, this preliminary study emphasizes the need for extending and confirming these data in order to properly prevent and treat individuals from this geographic area. Extraordinary hope for antiretroviral therapy in South Africa was observed in HIV-1 subtype C-infected patients who were co-infected with either Mycobacterium tuberculosis or HHV-8 [14,15].
Another study approved by the institutions and governments of Brazil and MZ on HHV-8 infection that includes individuals representing different risk-factors from the northern, central and southern regions of MZ is currently in progress. Owing to Brazilian experience in the fight against AIDS and KS [13] along with the results obtained from prior and present studies, we hope to provide information to the government of $\mathrm{MZ}$ regarding how to manage and control this emerging infectious disease.

\section{References}

1. Hengee U.R., Ruzicka T., Tyring S.K., Seeber S. Update on Kaposi’s sarcoma and other HHV-8 associated diseases. Part. 1: epidemiology, environmental predispositions, and therapy. Lancet Inf Dis 2002;2:281-92.

2. Szajerka T., Jablecki J. Kaposi’s sarcoma revisited. Aids Rev 2007;9:230-6.

3. UNAIDS. Report on Global AIDS Epidemic. UNAIDS, Geneva. 2006.

4. Dedicoat M., Newton R. Review of the distribution of Kaposi's sarcoma-associated herpesvirus (KSHV) in Africa in relation to the incidence of Kaposi's sarcoma. Br J Cancer 2003;88:1-3.

5. Sissolak G., Mayaud P. AIDS-related Kaposi's sarcoma: epidemiological, diagnostic, treatment and control aspects in sub-Saharan Africa. Tropical Medicine and International Health 2005;10:981-92.

6. Caterino-de-Araujo A., Carbone P.H.L., Martinelli F.L.B. et al. Absence of an association between the presence of HHV-8 antibodies and the development of Kaposi's sarcoma in HIV-1infected patients receiving antiretroviral therapy. AIDS 2000;14:1455-7.

7. Ministério da Saúde. Relatorio sobre a Revisão dos Dados da Vigilancia Epidemiológica do HIV - Ronda 2007, Maputo, 2008.

8. Silva J.M.K., Magri M.C., Jacob F., et al. Otimização e avaliação do ensaio de imunofluorescência indireta para pesquisa de anticorpos anti-herpesvírus humano 8 (HHV-8) usando sangue colhido em papel de filtro. Rev Inst Adolfo Lutz 2007;6:68-72.

9. Ministério da Saúde. Conselho de Ministros da República de Moçambique: Plano Estratégico Nacional de Combate ao HIVSIDA, PEN 2005-2009, Maputo, Novembro 2004.

10. Wojcicki J.M., Newton R., Urban M.I., et al. Low socioeconomic status and risk for infection with human herpesvirus 8 among HIV-1 negative, South African black cancer patients. Epidemiol Infect 2004;132:1191-7.

11. Mbulaiteye S.M., Biggar R.J., Pfeifer R.M., et al. Water, socioeconomic factors, and human herpesvirus 8 infection in Ugandan children and their mothers. J Acquir Immune Defic Syndr 2005;38:474-9.

12. Santos-Fortuna E., Caterino-de-Araujo A. Confirming shedding of human herpesvirus 8 in urine from Brazilian infected patients. J Clin Microbiol 2005;43:1008.

13. Caterino-de-Araujo A. The fight against Kaposi's sarcoma in AIDS - Lessons from Brazil. Southern African Journal of HIV Medicine 2007;26:53-4.

14. Cassol E., Page T., Mosam A., et al. Therapeutic response of HIV1 subtype $\mathrm{C}$ in African patients coinfected with either Mycobacterium tuberculosis or Human herpesvirus-8. J Infect Dis 2005;191:324-32.

15. Flanigan T.P., Campbell T., Harwell J., Kumarasamy N. The extraordinary hope of antiretroviral therapy in South Africa (even for patients with tuberculosis or Kaposi sarcoma!). J Infect Dis 2005;191:321-3. 